\title{
MLP ANN Condition Assessment Model of the Turbogenerator Shaft A6 HPP Đerdap 2
}

\author{
Dragoljub ILIĆ, Dragan MILOŠEVIĆ, Zoran JOVANOVIĆ, Milena CVJETKOVIĆ, Miroslav VULIĆ*
}

\begin{abstract}
This paper describes a model for estimating the condition of the shafts of turbines of the current generator in Hydropower plant Đerdap 2. For this purpose, an integral diagnostic approach was used. Based on the diagnostics of the condition of the shaft and the estimated lifetime, a multi-layer perceptron (MLP) based artificial neural network (ANN) is built, which is able to estimate the remaining lifespan of the turbine shaft. The MLP ANN model has not been made in this way on turbogenerators of hydroelectric power plant Đerdap 2 until now. The significance of this approach is that experiment brings about topology of ML ANN (number of neurons and layers) which is optimal for this model, training and testing. Results obtained from the neural network can be further used for decision-making about the moment of diagnosis or maintenance actions, as well as reducing stagnation and production losses.
\end{abstract}

Keywords: ANN (Artificial Neural Network); model; shaft; turbogenerator

\section{INTRODUCTION}

On the 863rd kilometer from the mouth of the Danube into the Black Sea, a river-flow hydroelectric power plant "Đerdap 2" was built, where 10 generators were installed in the period from 1985 to 2000 , each installed capacity of $27 \mathrm{MW}$. The basic parts of the turbo generator are shown in Fig. 1.

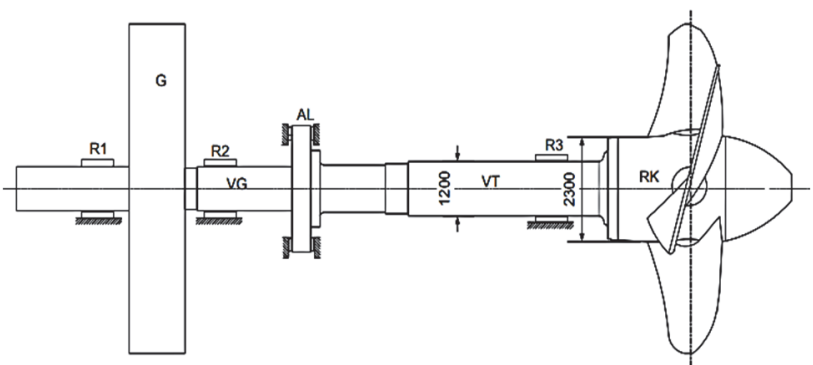

Figure 1 Basic generator parts $\mathrm{PL}$ - 15/826-G-750 (R1, R2 and R3 - radial bearings, $A L$ - axial bearing, $G$-Generator rotor,VG - generator shaft, VT turbine shaft, RK - operating circuit)

The failure to observe refers to the turbine shaft. The flange zone to the hub RK and the transient radius $\mathrm{R} 80$ are under the influence of leaking water through the shaft seal. The problem that needs to be solved is to evaluate the condition of the turbine shaft, based on the diagnostics of the state using non-invasive methods, and in periods of time when there is no delay, the application of diagnostics is not possible. Since budgets are always approximate and all known methods have their limitations and diagnostics in the form of monitoring is not available, it is necessary to rely on previous experiences, diagnostics, real and specific conditions of exploitation that are valid on the aggregates of HPP Đerdap 2 on the Danube.

A small number of failures, as well as a small number of diagnostic measurements due to the need of continuous operation of the turbogenerator, make this problem even more complex, and that is precisely the reason to approach the problem through MLP ANN.

Choosing a deep model encodes a very general belief that the function we want to learn should involve composition of several simpler functions [1]. On that basis, we believe that by applying MLP ANN it is possible to find an approximation of the state of the turbogenerator shaft.
The topic is important because predicting the state of the technical system of the turbogenerator shaft as a critical place in terms of reliability of the entire aggregate could save huge downtimes for diagnostics and select optimal diagnostic moments depending on the exploatation conditions.

The wider social significance is energy stability, which is achieved through planned maintenance and diagnostics actions and by avoiding unplanned downtime and failures that have occurred in the past on the A6 and other aggregates.

\subsection{Literature Review}

The ANN represents an artificial system based on mathematical models which is, in its structure, function and information processing, similar to the biological nervous systems and thus able to intelligently process the information simulating the biological intelligence [2]. ANNs are used for the purpose of diagnostics of the condition of the shafts on turbines and even within the monitoring [3] but in conditions where there is no continuous diagnostics or when it is not possible in an adequate manner, this limiting factor in the application of such models is impossible. Alternatives are found in reliability models that indicate the probability of faultless work, usually based on probability theory, Weibull's distribution, Monte Carlo simulations, etc [4]. Such models can be successfully used to optimize preventive maintenance on technical systems [5]. Some of the models aim to raise the reliability level of the technical system based on Fault Detection and Fault Tolerant Control [6], but this is associated with the existence of more failures and the ability to reliably monitor reliability parameters such as failure rates. However, when there is a diagnosis of the condition but which is occasional and insufficient, and when there is also a statistically low number of failures, (insufficient for the calculation of reliability parameters) due to the fact that congestion and production losses create an imperative as the longer work of a technical system such as a turbogenerator, model of turbogenerator shaft condition evaluation. The benefits of the use of neural networks exceed many times the work required to create them. In practice the longest stage of the process of 
creating the networks is the collection and preparation of source data [7].

Some of the researches (Pamucar, 2018), [8] show that ML ANNs are very effective for the implementation of intelligent decision support systems for route selection. ANN can also imitate the decision-making of transport support managers and provide actual routing advice to the driver in real-time traffic conditions. In this paper [*Pamucar], the ANFIS model can reproduce the decisions of dispatchers with great accuracy and even substitute decisions of transport support organs.

ML ANNs are also used to predict the maximum energy of photovoltaic modules (Stojcic, 2019), [9]. The prediction of used ANFIS (Adaptive Neuro Fuzzy Inference System) model should facilitate work in planning production and consumption, system management and economic analysis. The model represents a simple solution that yields better results than mathematical model.

\subsection{Proposed Method Advantages}

ML ANN is a form of artificial intelligence that can be further developed and applied widely to other, similar technical systems. It is a reliable fast prediction method feasible for computer processing and simulations but it is particularly feasible because under the given operating conditions clear functional interdependence could not be determined between operating modes (0-5 MW, 5-10 MW, etc.) relative to service life and shaft dynamic durability as a key place for reliability of the whole system (aggregate). Therefore, this method is feasible to take into account factors that are unknown for the given conditions of exploitation and to disintegrate them and include them into the model. The ANN method has already been used in shaft diagnostics but rarely ML. ML ANNs could determine more complex interfaces such as this one, where many factors affect the operating modes interdependence and dynamic durability.

The model is not based on monitoring, which is an advantage when it is not workable, especially when there is a willingness for continuous operation of the technical system and when production losses are high due to downtime which is necessary for more complete diagnostics.

\section{TURBOGENERATOR SHAFT FAILURE PROBLEM}

During exploitation in the flange zone, microcracks first appear, which eventually spread and ultimately lead to fracture. Four flange conditions were identified:

- new state,

- creation of initial cracks,

- growth of initial cracks,

- final breakdown phase.

Table 1 Values of Coefficient of dynamic durability

\begin{tabular}{|c|c|}
\hline Shaft flange state & Coefficient of dynamic durability $-C_{\mathrm{di}}$ \\
\hline New state & 1,00 \\
\hline Creation of initial cracks & 0,66 \\
\hline Growth of initial cracks & 0,33 \\
\hline Final breakdown phase & 0,00 \\
\hline
\end{tabular}

Based on these four conditions, the coefficient $C_{\mathrm{di}}$ was introduced, which pertains to the dynamic durability of the flange which lies in the range of 1 - completely new state to 0 - shaft breakdown (Tab. 2).

Interstates are linearly interpolated values based on the analogy with the Woler diagram.

Diagnostics of turbine conditions that were made during the exploitation period are:

- vibrodiagnostics,

- magnetic particle diagnostics,

- penetrant diagnostics,

- visual diagnostics,

- ultrasound diagnostics.

As input variables for the neuron network, the following were used:

- operating days of the turbogenerator,

- $\quad$ operating modes (5 operating modes),

- coefficient of dynamic durability of the shaft at the beginning of the calendar year.

The output of the neural network is the coefficient of dynamic durability of the shaft at the end of the calendar year.

ANN simulates the work of the shaft and the movement of the dynamic durability coefficient at the beginning and end of each calendar year and depending on the mode of operation and the produced electricity. This can also result in the failure of the shaft, based on operating modes.

\section{NETWORK DESIGN}

Selection of hidden neurons using the neural networks is one of the major problems in the field of Artificial Neural Network. Overtraining is the same as the issue of overfitting data. Overtraining arises because the network matches the data so closely as to lose its generalization ability over the test data [10].

The Input Layer is a layer which communicates with the external environment. It also represents the condition for which we are training the neural network [11].

In this case, the number of defined input neurons is 7 which are defined based on 7 input variables:

- operating days of the turbogenerator,

- operating mode 0 - $5 \mathrm{MW}$,

- operating mode 5 - $10 \mathrm{MW}$,

- operating mode $10-15 \mathrm{MW}$,

- operating mode $15-20 \mathrm{MW}$,

operating mode $20-27 \mathrm{MW}$,

- coefficient of dynamic durability of the shaft $C_{\mathrm{di}}$ at the beginning of the calendar year.

The Output layer of the neural network is what actually presents a pattern to the external environment. The number of output neurons should be directly related to the type of the work that the neural network is to perform [11]. The number of output neurons is one and it designates only one variable, which is the coefficient of dynamic durability of the shaft $C_{\mathrm{di}}$ at the end of calendar year.

The Hidden layer of the neural network is the intermediate layer between Input and Output layer. Hidden layer consists of hidden nodes (or perceptrons). Hidden nodes or hidden neurons are the neurons that are neither in the input layer nor the output layer [11].

Single-layer networks have just one layer of active units. The greatest number of ANNs has one hidden layer though there may be more of them $[12,13]$. 
Modern ANNs have a parallel-distributed architecture. They consist of a large number of neurons distributed in several layers. Each ANN must have three layers: input layer, hidden layer and output layer.

The standard multilayer perceptron (MLP) is a cascade of single-layer perceptrons [14].

Although a single hidden layer is optimal for some functions, there are others for which a single-hidden-layersolution is very inefficient compared to solutions with more layers [14].

Determination of the number of layers ie the depth of the net as well as the number of neurons in each of the layers, the width of the network, is exactly the most difficult task and it is necessary to have a certain tactic of approaching this solution. First, this certainly involves experimentation and even some kind of intuitive search. Here we relied on a systematic way to find the best solution by finding the most optimal number of neurons for a singlecore network, then added another layer and tried out training and tests with a higher number of neurons and chose the one with the best results. After that, a new layer is added again until the results of training and testing are not reduced, which prevents overfitting through ANN complexity control.

\section{NETWORK TRAINING AND TESTING METHODS}

The learning problem in neural networks is formulated in terms of the minimization of a loss function, $f$. This function is, in general, composed of an error and a regularization terms. The error term evaluates how a neural network fits the data set. On the other hand, the regularization term is used to prevent overfitting, by controlling the effective complexity of the neural network.

Quasi-Newton algorithm was selected as learning algorithm. Then, the quasi-Newton formula can be expressed as:

$w^{(i+1)}=w^{(i)}-\left(\boldsymbol{G}^{(i)} \cdot g^{(i)}\right) \cdot \eta^{(i)} \quad i=0,1, \ldots, n$

The activity diagram of the quasi-Newton training process is illustrated bellow.

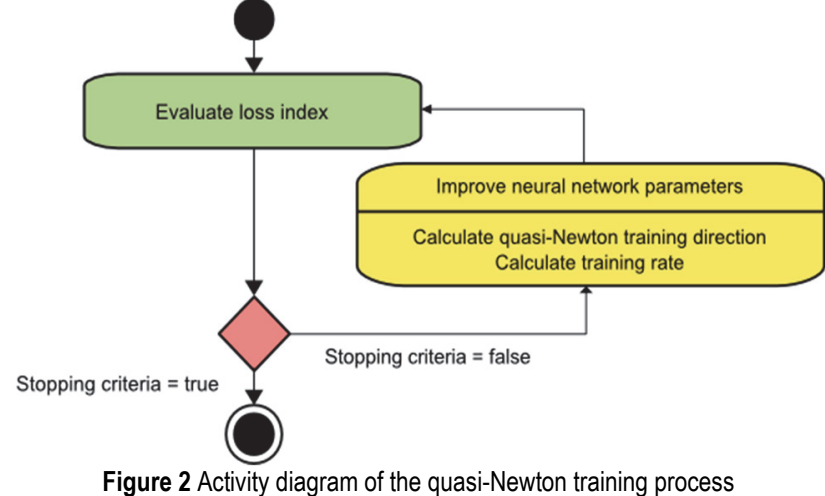

This is the default method to use in most cases: It is faster than gradient descent and conjugate gradient, and the exact Hessian does not need to be computed and inverted because quasi-Newton method is to approximate the inverse Hessian by another matrix $\boldsymbol{G}$ using only the first partial derivatives of the loss function.

\section{DATA SET}

The data set contains the information for creating the predictive model. It comprises a data matrix in which columns represent variables and rows represent instances.

Variables in a data set can be of two types:

- the inputs will be the independent variables (7 variables),

- $\quad$ the targets will be the dependent variables (1 variable).

Additionally, instances are:

- training instances, which are used to construct the model,

- $\quad$ selection instances, which are used for selecting the optimal order,

- testing instances, which are used to validate the functioning of the model; unused instances, which are not used at all.

Tab. 1 shows a preview of the data matrix contained Here, the number of variables is 8 , and the number of instances is 186 .

\begin{tabular}{|c|c|c|c|c|c|c|c|c|}
\hline & Days & $0-5 \mathrm{MW}$ & $5-10 \mathrm{MW}$ & $10-15 \mathrm{MW}$ & $15-20 \mathrm{MW}$ & $20-27 \mathrm{MW}$ & Input & Outnut \\
\hline 1 & 264 & 0,958466 & 1,23861 & 4,34891 & 42,8342 & 121,139 & 0,984163 & 0,962268 \\
\hline 2 & 629 & 0,201319 & 0,681705 & 4,45211 & 64,2088 & 112,806 & 0,962268 & 0,940372 \\
\hline & & & & & & & & \\
\hline 186 & 2283 & 0,406509 & 0,857552 & 4,69883 & 31,9542 & 102,494 & 0,754516 & 0,715269 \\
\hline
\end{tabular}

Table 3 Data statistics

\begin{tabular}{|c|c|c|c|c|}
\hline & Minimum & Maximum & Mean & Deviation \\
\hline Days & 0 & 7934 & 2797,04 & 2154,86 \\
\hline $0-5 \mathrm{MW}$ & 0,189208 & 2,18625 & 0,612196 & 0,472398 \\
\hline $5-10 \mathrm{MW}$ & 0,414203 & 1,57913 & 0,943866 & 0,264452 \\
\hline $10-15 \mathrm{MW}$ & 0,643878 & 6,00288 & 3,43495 & 1,12012 \\
\hline $15-20 \mathrm{MW}$ & 8,91366 & 66,0534 & 39,1452 & 11,4972 \\
\hline $20-27 \mathrm{MW}$ & 54,8773 & 171,609 & 108,868 & 16,7215 \\
\hline Input & 0,0092045 & 1 & 0,700621 & 0,234216 \\
\hline Output & 0 & 0,981514 & 0,663399 & 0,23797 \\
\hline
\end{tabular}

The total number of instances is 186 . The number of training instances is $112(60.2 \%)$, the number of selection instances is $37(19.9 \%)$, the number of testing instances is $37(19.9 \%)$.
Tab. 2 shows the minimums, maximums, means and standard deviations of all the variables in the data set.This is a very important data because outputs are obtained approximations that are valid only if all inputs are within the limits between the maximum and the minimum. Experience shows that good results are not obtained if these limit values of input parameters are exceeded. By collecting data from the work of the power plant, the range of the boundaries is extremely wide and provides plenty of room for testing various inputs since the neuron network is formed and trained. 


\section{DESIGNATION OF THE STRUCTURE OF NEURON NETWORK}

Starting from layer one, model selection is applied to find a neural network with a topology that optimizes the loss on data. After that, a new layer is applied and the procedure is repeated in order to find the number of neurons in the new layer with the least loss on data. Order selection algorithms and input selection algorithms. Order selection algorithm is used to find the optimal number of hidden neurons in the network. This method starts with the minimum order and adds a given number of perceptrons in each iteration. The minimum number of neurons is one, and the maximum up to 50 including step one. Each is performed in three attempts in order to find the least loss data where the selection loss goal equals zero. The maximum number of iterations is 1000 .

For all hidden neurons, the Hyperbolic Tangent activation function was used, and for output neuron, the Linear function was used. Normalized squared error (NSE) was used as Error method. The aforementioned QuasiNewton method was used as training algorithm. Minimum selection loss was used as selection criteria.

Tab. 3 displays results after each iteration of the order selection algorithm.

Table 4 Order selection algorithm results

\begin{tabular}{|c|c|c|c|}
\hline Number of hidden layer & $\begin{array}{c}\text { Structure of neuron network } \\
\text { input layer : hidden layers : output layers }\end{array}$ & Minimum training loss & Minimum selection loss \\
\hline 1 & $7: 13: 1$ & 0,0034472 & 0,00600015 \\
\hline 2 & $\mathbf{7}: 13: 10: \mathbf{1}$ & 0,0037146 & 0,00518826 \\
\hline 3 & $\mathbf{7}: 13: 10: 7: \mathbf{1}$ & 0,003838996 & 0,00401436 \\
\hline 4 & $\mathbf{7}: 13: 10: 7: 2: \mathbf{1}$ & 0,00386726 & 0,0038546 \\
\hline
\end{tabular}

The structure of neuron network obtained at the end is displayed in Fig. 3.

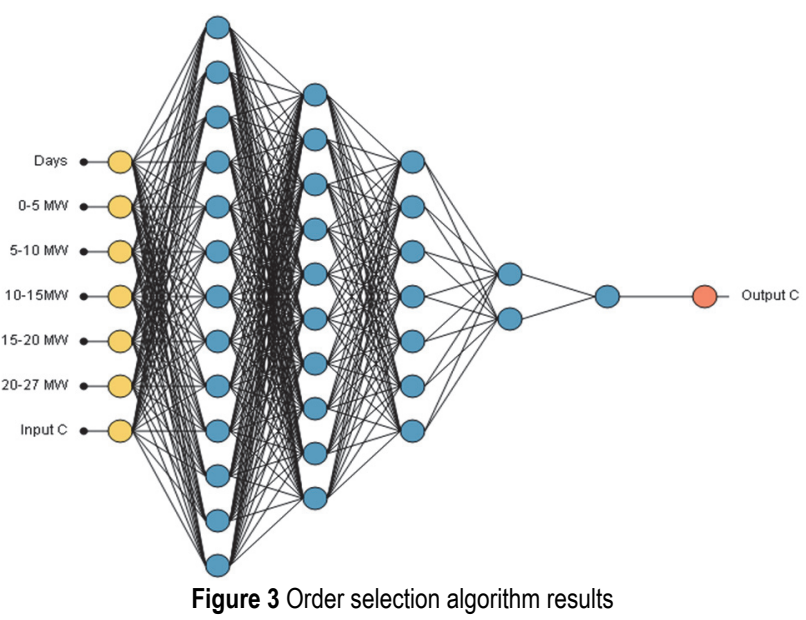

\section{NETWORK TRAINING}

After that, the series of trainings was accessed. Training strategy implied training in multiple series because the data set is relatively small, and in order to obtain better results. The training strategy is applied to the neural network in order to obtain the best possible loss.

Training rate method used to calculate the step for the quasi-Newton training direction is Brent Method. Training accuracy depends on the training rate tolerance which is in range from 0,05 to 0,0005 each time with 5 times reduced value, in order to obtain greater precision after 5 training series.

Minimum parameters increment norm is 0 at which training stops. Minimum loss increase (minimum loss improvement between two successive iteration), goal value for the loss and Gradient norm goal are 0. Maximum number of iterations at which the selection loss increases is 100. Maximum iterations number is $10000 \mathrm{M}$. The minimum training loss is observed as criteria. 5 .

Results of the last training series are displayed in Tab.

Table 5 Training results
\begin{tabular}{|c|c|}
\hline Parameter & Value \\
\hline Final training loss & 0,181 \\
\hline Final selection loss & 0,00363 \\
\hline Iterations number & 4448 \\
\hline
\end{tabular}

\section{RESULTS}

Upon completion of the training process of the neuron network, the possibility of approximating the assessment of the state of the turbogenerator A6 HPP Đerdap 2 has been created based on the load defined by the working regimes, i.e., annual electricity production.

Fig. 4 displays the movement of the coefficient of dynamic durability of the shaft $C_{\mathrm{di}}$ depending on the production of electric power. Production from database is given as the first example.

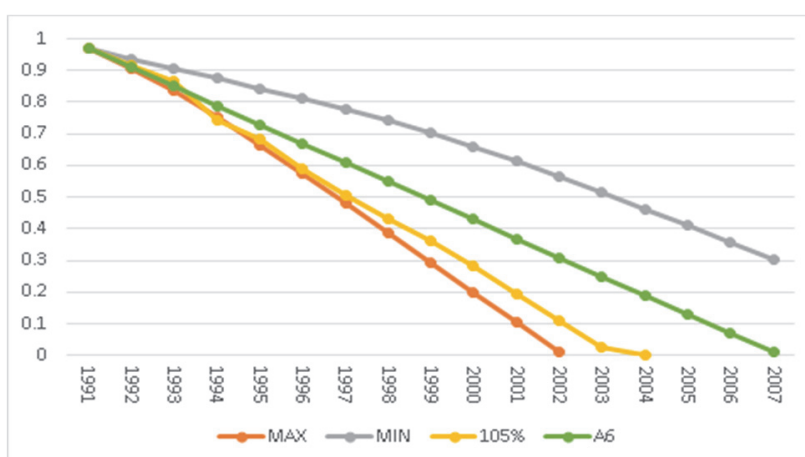

Figure 4 Changes of the coefficient of the dynamic durability of the shaft $C_{\mathrm{di}}$ for different scenarios of the electric power production of the turbogenerator $A 6$

The first scenario that the neural network examined was the case of the largest recorded production taken from a database of an average of 176,59 MW over one year and associated operating regimes $(0,36 \mathrm{MW}, 0,78 \mathrm{MW}, 4,73$ MW, 49,89 MW, 120,83 MW). Under these conditions, the shaft breakdown would occur in 2003, instead of 2007.

The second scenario is the increase by $5 \%$ of each annual production from the base, proportionally by operating regimes. This production would lead to shaft failure during 2004. 
The third scenario implies the minimum recorded production in the database during each year of scenarios of 120,96 MW during one year and attributable operating regimes $(0,25 \mathrm{MW}, 0,54 \mathrm{MW}, 3,02 \mathrm{MW}, 35,06 \mathrm{MW}, 82,10$ MW). This exploitation scenario lead to the values of coefficient of dynamic durability of the shaft $C_{\mathrm{di}}=$ 0,254862826 at the end of 2007 .

These scenarios also imply the total amount of produced energy as a result, which is displayed in Fig. 5.

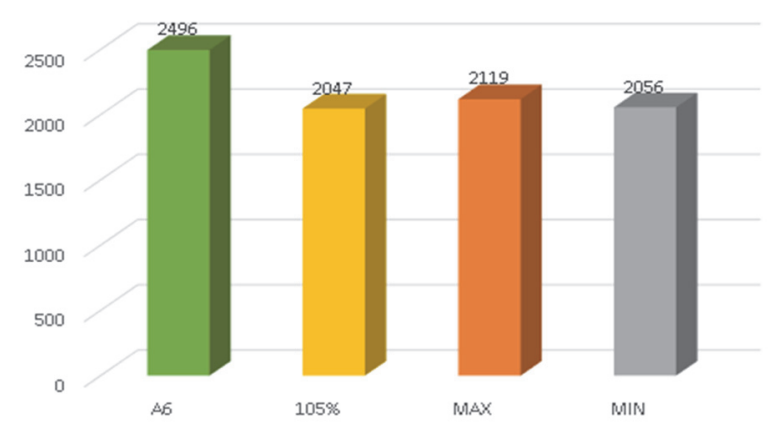

Figure 5 Total productions of electric power for different exploitation scenarios (MW)

An important fact is that the minimal production scenario is such that full exploitation has not occurred and that the produced amount of electrical energy is higher than it is visible on the graphics. It is clear that the increase in electricity generation considerably shortens the lifetime of the turbogenerator shaft and that, consequently, the amount of electricity produced is lower. Dynamic impacts on the impeller lead to the formation of microcracks and their accelerated propagation, and the final break of the shaft is much faster during the more intense turbogenerator exploitation.

However, due to the faster exploitation of turbogenerators, it is certainly also due to the fact that these quantities of energy can be produced in a shorter time period, so it is representative to look at the average annual outputs illustrated in Fig. 6.

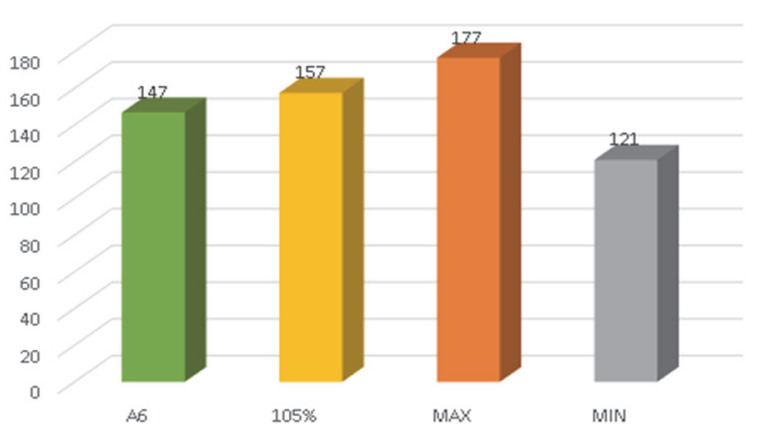

Figure 6 Annual productions of electric power for different exploitation scenarios (MW)

Fig. 6 reveals that a higher level of exploitation results in a higher average annual production, although overall production is lower when the exploitation level is higher. The neural network model allows production planning to be in accordance with energy needs and appreciating the level of turbine generator shaft consumption.

Concerning classical approaches such as the application of a Wöhler diagram (curve) that is suitable for test conditions or finite-element method, which is often used [15], the ML ANN method takes into account the complex exploitation conditions for a given technical system at the point of installation and allows more reliable predictions based on such conditions.

Even after the onset of fatigue cracks and turbine failure, carry out extensive fault analysis and numerical analysis [16] which are conducting to further suggestions for failure prevention, Nevertheless, the ML ANN method used in this paper gives much faster estimates of the time required to conduct, and also provides prediction of turbine condition.

It is especially capable to accept predefined workloads. Compared to the application of ML ANN for turbine shaft diagnostics, which is presented in [3], our model does not deal with the current condition estimation based on parameters and gives the state predictions, based on the operating regimes for which production is most directly interested. The model also works in conditions with fewer diagnostic measurements and is adaptable to the new measurements if available.

\section{CONCLUSION}

The novelty of this model is an integrated approach that could connect all types of diagnostics into the unique whole and assess shaft condition by non-destructive methods and with that reduce unnecessary downtime. Also, the novelty of this model is in conditions where there is a statistically small number of failures and also the application of classic models does not make sense so by interpolating diagnostic values a sufficient number of data for training and testing of ANN was obtained.

The significance of the approach is in experimentally reaching the maximum capability for loading the system as well as all possible working regimes and predicting the state of the technical system afterward. This enables easy, fast and efficient planning of production based on concrete production conditions, and optimum exploitation of the technical system during its lifetime.

The result of the model of the assessment of the state of the turbogenerator shaft A6 based on MLP NN enables wide possibilities. The rapid possibility of forecasting the turbogenerator shaft consumption based on different scenarios of electricity generation gives the potential to use resources optimally in relation to energy needs. A special advantage is the possibility to momentarily obtain the assessment of the condition of the shaft and temporal distance from the possible failure of the shaft flange due to water impacts, without the loss of time, calculation and approximation of real conditions of exploitation. One of the more serious advantages is the possibility to reduce the stoppages and thus losses, of numerous and unnecessary diagnostics of the shaft state, and to optimize the number of diagnostics checks. The model was made in such a way that new state diagnostics may adjust the values of training data sets and perform new machine learning of neuron network on an already established topology and thus obtain information of even greater quality, if the exploitation conditions and properties of technical system were to change. This makes the model a good foundation for further applications in the field of turbogenerator shaft failure. 
Further research could go in the next directions:

model application to similar systems and generalization of conclusions in terms of generalization of solutions,

creation of Decision Support Systems to assist decision-makers (on the optimal timing of actions of maintenance and diagnostics),

development of artificial intelligence form that could be based on the prediction of the state of the system and other production requirements to manage the operating modes and even the entire production in the future,

software development as a didactic training tool for maintainers and students to create a wider picture of the consequences of applying certain production strategies to the reliability and operation of the turbogenerator technical system (as the most important part of a hydroelectric power plant).

\section{REFERENCES}

[1] Goodfellow, I., Bengio, Y., \& Courville, A. (2016). Deep Learning (Adaptive Computation and Machine Learning series). Cambridge, Massachusetts: A Bradford Book.

[2] Peko, I., Nedic, B., Djordjevic, A., \& Veza, I. (2018). Modelling of Kerf Width in Plasma Jet Metal Cutting Process using ANN Approach. Technical gazette, 25(2), 401-406. https://doi.org/10.17559/TV-20161024093323

[3] Rahmoune, M. B., Hafaifa, A., Abdellah, K., \& Chen, X. Q. (2017). Monitoring of high-speed shaft of gas turbine using artificial neural networks: Predictive model application. Diagnostyka, 18(4), 3-10.

[4] Kucora, I., Radovanovic, L., Milosevic, D., Vulovic, S., Kovacevic, M., Otic, G., \& Adamovic, Z. (2017). Increasing the safety of power plants using a new model of reliability. Energy Sources, Part B: Economics, Planning, and Policy, 12(5), 460-469. https://doi.org/10.1080/15567249.2016.1185481

[5] Milosevic, D., Janjic, N., Vulovic, V., \& Adamovic, Z. (2016). Optimisation of Preventive Maintenance of Lubrication Subsystem by Reliability Simulation Model of V46-6 Engine. Journal of the Balkan Tribological Association, 22(1), 419-432.

[6] Habibi, H., Howard, I., \& Simani, S. (2019). Reliability improvement of wind turbine power generation using modelbased fault detection and fault tolerant control: A review. Renewable Energy, 135, 877-896. https://doi.org/10.1016/.j.renene.2018.12.066

[7] Rojek, I., Kowal, M., \& Stoic, A. (2017). Predictive compensation of thermal deformations of ball screws in CNC machines using neural networks. Technical gazette, 24(6), 1697-1703. https://doi.org/10.17559/TV-20161207171012

[8] Pamucar, D. \& Cirovic, G. (2018). Vehicle route selection with an adaptive neuro fuzzy inference system in uncertainty conditions. Decision Making: Applications in Management and Engineering, 1(1), 13-37. https://doi.org/10.31181/dmame180113p

[9] Stojcic, M., Stjepanovic, A., \& Stjepanovic, D. (2019). ANFIS model for the prediction of generated electricity of photo voltaic modules. Decision Making: Applications in Management and Engineering, 2(1), 35-48. https://doi.org/10.31181/dmame1901035s

[10] Panchal, S. F. \& Panchal, M. (2014). Review on Methods of Selecting Number of Hidden Nodes in Artificial Neural Network. International Journal of Computer Science and Mobile Computing, 24(6), 455-464.

[11] Karsoliya, S. (2012). Approximating Number of Hidden layer neurons in Multiple Hidden Layer BPNN Architecture.
International Journal of Engineering Trends and Technology, 3(6), 714-717.

[12] Elangovan, K., Sathiya Narayanan, C., \& Narayanasamy, R. (2010). Modeling and forming limit diagram of perforated commercial pure aluminium sheets using artificial neural network. Computational Materials Science, 47(4), 10721078. https://doi.org/10.1016/j.commatsci.2009.12.016

[13] Miljković, Z., Bojović, B., \& Babić, B. (2010). Application of artificial neural network and fractals in biomedical materials surface behaviour prediction. Tehnika - Novi materijali, 19(4), 5-14.

[14] Reed, R. \& Marks II, J. R. (1999). Neural Smithing: Supervised Learning in Feedforward Artificial Neural Networks. Cambridge, Massachusetts: A Bradford Book. https://doi.org/10.7551/mitpress/4937.001.0001

[15] Dadashi, A., Poursaeidi, E., \& Amini, H. (2016). Stress analysis of steam turbine shaft using finite element method. ICESCON02, Istanbul, Turkey.

[16] Momcilovic, D., Odanovic, Z., Mitrovic, R., Atanasovska, I., \& Vuherer, T. (2012). Failure analysis of hydraulic turbine shaft. Engineering Failure Analysis, 20(1), 54-66. https://doi.org/10.1016/j.engfailanal.2011.10.006

\section{Contact information: \\ Dragoljub ILIĆ, PhD Student \\ Aviation Academy, \\ Bulevar vojvode Bojovića 2, 11000 Belgrade, Serbia \\ E-mail: ilicdragoljub57@gmail.com}

Dragan MILOŠEVIĆ, Associate Professor

University Business Academy in Novi Sad

Faculity of Economics and Engineering Management in Novi Sad,

Cvećarska 2, 21000 Novi Sad, Serbia

E-mail: prof.dragan.milosevic@gmail.com

Zoran JOVANOVIĆ, Associate Professor

College "Dositej",

Bulevar vojvode Putnika 7, 11000 Belgrade, Serbia

E-mail: zojo30@yahoo.com

\section{Milena CVJETKOVIĆ, Assistant Professor}

College "Dositej",

Bulevar vojvode Putnika 7, 11000 Belgrade, Serbia

E-mail: cvjetkovicm@gmail.com

Miroslav VULIĆ, Teaching Assistant

(Corresponding author)

University Business Academy in Novi Sad,

Faculty of Economics and Engineering Management in Novi Sad,

Cvećarska 2, 21000 Novi Sad, Serbia

E-mail: miroslavvulic@live.com 